\title{
Characterization of a Liquid Crystal Stabilized Pharmaceutical Oil-in-Water Emulsion Optimized for Skin Delivery
}

\author{
Melinda J. Sutton'1, David W. Osborne ${ }^{1,2}$, Kevin Dahl ${ }^{3}$, Victoria Bax ${ }^{1}$, G. Alan Schick ${ }^{1}$ \\ ${ }^{1}$ Department of Chemistry, Missouri State University, Springfield, MO, USA \\ ${ }^{2}$ Dow Development Institute, Petaluma, CA, USA \\ ${ }^{3} \mathrm{KBI}$ Biopharma, Boulder, CO, USA \\ Email: david.wade.osborne@gmail.com
}

How to cite this paper: Sutton, M.J., Osborne, D.W., Dahl, K., Bax, V. and Schick, G.A. (2018) Characterization of a Liquid Crystal Stabilized Pharmaceutical Oil-inWater Emulsion Optimized for Skin Delivery. Journal of Cosmetics, Dermatological Sciences and Applications, 8, 207-217. https://doi.org/10.4236/jcdsa.2018.84022

Received: October 6, 2018

Accepted: November 13, 2018

Published: November 16, 2018

Copyright ( 92018 by authors and Scientific Research Publishing Inc. This work is licensed under the Creative Commons Attribution International License (CC BY 4.0).

http://creativecommons.org/licenses/by/4.0/

\section{cc) (i) Open Access}

\begin{abstract}
A moisturizing cream containing $25 \mathrm{wt} \%$ of an organic solvent, diethylene glycol monoethyl ether (DEGEE), is observed to be stabilized by an emulsifying wax blend of cetearyl alcohol, dicetyl phosphate, and ceteth-10 phosphate (tradename Crodafos CES). Polarized light microscopy indicates that the Crodafos CES helps to produce a liquid-crystal stabilized oil-in-water emulsion, which is physically stable for months under accelerated aging conditions and chemically stable over the full topical $\mathrm{pH}$ range of 3.5 to 9. Emulsion globule size in the cream is observed to be dependent on the degree of emulsifying wax neutralization, with the globule size decreasing with increasing cream $\mathrm{pH}$. The superior solubilizing properties of DEGEE combined with the full $\mathrm{pH}$ range and liquid-crystal stabilizing properties of the Crodafos CES give this formulation the potential for a wide range of applications in the topical delivery of active pharmaceutical ingredients.
\end{abstract}

\section{Keywords}

Pharmaceutical Emulsion, Skin Delivery, Liquid Crystal Stabilized Emulsion, Polarized Light Microscopy, Topical Formulation

\section{Introduction}

Diethylene glycol monoethyl ether (DEGEE) is a compendial excipient that is being used increasingly as a solvent in FDA approved topical pharmaceutical products [1]. Despite being water miscible, DEGEE is a good solvent for lipophilic materials such as skin oils. Topical gel products containing significant 
amounts of DEGEE ( $\geq 25 \% \mathrm{w} / \mathrm{w}$ ) have been developed having good skin feel when applied to anatomical sites rich in sebum, such as the face [1]. However, the advantages of formulating with DEGEE for application to oily skin sites quickly becomes a disadvantage when formulating for skin conditions that are characterized by "dry skin". For these dermatological applications, a moisturizing formulation such as an oil-in-water cream or lotion is required [2]. Due to DEGEE being such a good solvent for lipophilic materials, the oil phase of the emulsion tends to dissolve when this solvent is added to a traditional oil-in-water emulsion. Although this property has made DEGEE a preferred solvent for formulating microemulsions and nanoemulsions [3], development of an oil-in-water emulsion that contains a significant amount of DEGEE represents a significant challenge for the topical dermatological product formulation scientist.

Emulsions having liquid crystals present at the oil-water interface have been described as providing better moisturization, greater physical stability, and improved controlled release of pharmaceutical actives when compared with traditional two-liquid emulsions [4] [5]. It was noted in a patent application that $25 \%$ DEGEE could be incorporated into a moisturizing cream that used an anionic emulsifying wax marketed by Croda (tradename Crodafos CES) [6]. This anionic emulsifying wax, a blend of cetearyl alcohol, dicetyl phosphate, and ceteth-10 phosphate, is commonly used to suspend colloidal sunscreens and has been found to be well tolerated when applied topically [7]. It is also known to form liquid crystal stabilized emulsions that can be adjusted to a wide range of $\mathrm{pH}$ values [7]. Since topically applied actives typically penetrate human skin best when the drug is non-ionized [8], optimum skin penetration would be achievable for a given active in this cream simply by adjusting the $\mathrm{pH}$ of the cream based on the dissociation constant of the active.

This study uses polarized light microscopy to confirm that the cream is a liquid crystal stabilized emulsion over the range of $\mathrm{pH}$ values from 3.5 to 9 . Additionally, the effects of $\mathrm{pH}$ on the physical stability of the cream and globule size distribution in this oil-in-water emulsion are characterized. Since this cream contains $25 \%(\mathrm{w} / \mathrm{w})$ of the solvent DEGEE, it should be able to solubilize a wide range of pharmaceutical actives.

\section{Materials and Methods}

A series of 300-g cream batches were prepared for stability, $\mathrm{pH}$, and Microscopic View determination [9]. A specified amount of deionized water was weighed into a $150-\mathrm{mL}$ beaker. $1.00 \mathrm{~N} \mathrm{NaOH}$ (aq) was then added by weight to the beaker until the combined amount of water phase reached $149.25 \mathrm{~g}$. This water phase was then transferred into a $250-\mathrm{mL}$ Wheaton bottle, tightly capped and placed in an $85^{\circ} \mathrm{C}$ oven until being transferred to a warm $400-\mathrm{mL}$ beaker for processing of the cream.

The composition of the oil phase is given in Table 1. To prepare the oil phase, the semisolid, oily liquid, and wax pellets were combined in a beaker, melted by heating in the $85^{\circ} \mathrm{C}$ oven, and quickly stirred before slowly adding the mixture 
Table 1. Compositions of the water, oil, and DEGEE phases of the cream.

\begin{tabular}{ccc}
\hline Ingredient & Tradename & Wt $\%$ \\
\hline Water & & \\
$1.00 \mathrm{NNaOH}(a q)$ & & $49.75^{*}$ \\
Oil Phase & & \\
White Petrolatum USP & Crodamol IPP & 5.0 \\
Isopropyl Palmitate & Crodafos CES & 10.0 \\
Emulsifier blend & & \\
(cetearyl alcohol, dicetyl phosphate, and ceteth-10 phosphate) & & 25.0 \\
DEGEE Phase & Transcutol P & 2.0 \\
Diethylene Glycol Monoethyl Ether & & 0.20 \\
Methyl Paraben & & 0.05 \\
Propyl Paraben & & 100 \\
Total & &
\end{tabular}

${ }^{*}$ The water phase was made for specific $\mathrm{pH}$ by adding a measured mass of $1.00 \mathrm{~N} \mathrm{NaOH} \mathrm{(aq)} \mathrm{and} \mathrm{diluting}$ with water to a combined total of $49.75 \mathrm{wt} \%$ of the cream (see also Figure 1).

to the heated water phase under constant mixing with an IKA Eurostar power control viscosity propeller blade operating at $455 \mathrm{rpm}$. The mixing continued until the emulsion cooled to below $50^{\circ} \mathrm{C}(15-20$ minutes). As soon as the oil phase was completely transferred, a room-temperature DEGEE phase containing dissolved parabens (Table 1) was immediately transferred into the warm water and oil emulsion. The mixing speed remained at $455 \mathrm{rpm}$ until the cream cooled to $46^{\circ} \mathrm{C}-49^{\circ} \mathrm{C}$. At this temperature the emulsion was fully formed, the mixer was turned off, and the cream was poured into glass jars for storage.

Within three days of preparation, a 30-g sample of cream was placed in a centrifuge tube and separated by centrifugation using a Sorvall RC-58 Refrigerated Superspeed centrifuge $\left(21^{\circ} \mathrm{C}-23^{\circ} \mathrm{C}\right)$ equipped with a $5 \mathrm{~S}-34$ rotor operating at $20,000 \mathrm{rpm}(47,870 \mathrm{~g})$ for 2 hours. The separated aqueous phase was carefully removed with a glass pipette and weighed in a capped test tube. The $\mathrm{pH}$ was determined using a Mettler Toledo InLab Flex-Micro pH combination electrode. Additional samples (approximately $60 \mathrm{~g}$ ) of each cream were placed in jars and stored in either a $40^{\circ} \mathrm{C}$ oven or in a laboratory at ambient temperature $\left(22^{\circ} \mathrm{C}\right.$ $26^{\circ} \mathrm{C}$ ). At the ends of 1 -month (28 days) and 2-month (56 days) periods, $30-\mathrm{g}$ samples from each of the storage conditions were centrifuged, and the $\mathrm{pH}$ values of the water phases were determined.

Microscopic View testing was performed using an Amscope PZ300 Series polarized light microscope fitted with a $6 \mathrm{~V} / 30 \mathrm{~W}$ halogen bulb (Philips 5761). The Infinity Plan achromatic objective had $4 \times$ magnification, a numerical aperture of 0.10 , and a working distance of $7.8 \mathrm{~mm}$. Temperature was controlled to within $0.001^{\circ} \mathrm{C}$ using an Instec TS $102 \mathrm{~W}$ thermal stage.

A Malvern Morphologi G3SE-ID automated imaging system was used to de- 
termine globule sizes in the same creams evaluated by Microscopic View testing $(\mathrm{pH}$ values ranging from 4.4 to 9.2). Cream samples for globule-size analysis were prepared by taking a small aliquot of the cream and placing it in the middle of a cleaned microscope slide. A second microscope slide was placed on top of the sample, and the resulting "sandwich" was placed in a device designed to provide a $25-\mu \mathrm{m}$ thick cell. The device used an FTIR cell (BioTools, Inc., Jupiter FL, USA) consisting of top and bottom plates with four threaded posts and nuts. A $25-\mu \mathrm{m}$ spacer was used to control the thickness of the samples, and the nuts were tightened by hand in a torque pattern until significant resistance was encountered. The prepared sample was then removed and placed into a slide holder for image analysis.

Each sample was investigated using a $20 \times$ magnification method and focus was set manually. Individual aliquots were measured in triplicate by selection of three distinct regions of the sample, each encompassing a $1.5 \mathrm{~mm}$ radius circular area. The system was set up in a reduced angle-of-illumination geometry by controlling the bottom aperture, which has the effect of increasing the contrast of the globule centers versus the edge regions. The threshold of the image analysis software was set to include pixels with intensity greater than or equal to 180 , removing the darker interglobule regions from the analysis. Particles with a non-circular morphology were removed from the analysis through the use of a Solidity filter (solidity $<0.950$ ). Solidity is defined as the particle area divided by the Convex Hull Area of the particle, and removes particles with significant dead area. Volume-weighted (mass-weighted) particle size distributions are reported as averages of three measurements.

\section{Results}

The formulation composed according to Table 1 produced a smooth, white homogeneous cream that exhibited good physical stability. Before $\mathrm{pH}$ adjustment by sodium hydroxide, the $\mathrm{pH}$ of the separated water phase was 1.78 . Adding increasing amounts of $1.0-\mathrm{N} \mathrm{NaOH}(\mathrm{aq})$ allowed the cream to be formulated over the full topical $\mathrm{pH}$ range of 3.5 to 9 as shown in Figure 1. An inflection point in the $\mathrm{pH}$ curve that occurs between $\mathrm{pH} 5.5$ and 7.5 will make it difficult to target a specific cream $\mathrm{pH}$ in this range because relatively small differences in the amounts of added sodium hydroxide could result in half unit swings in the $\mathrm{pH}$ of the finished product cream. It is anticipated that reasonably tight $\mathrm{pH}$ specifications could be maintained for the formulation below $\mathrm{pH} 5.5$ and above $\mathrm{pH}$ 7.5. It should be noted that addition of a pharmaceutical active ingredient, especially at amounts above $1 \%-2 \%$ could shift this $\mathrm{pH}$ profile. Thus, this $\mathrm{pH}$ characterization of the specific cream base is only a starting point for development of a cream containing a pharmaceutical active.

Table 2 shows the $\mathrm{pH}$ value of the separated water phase of the creams for both ambient and accelerated $\left(40^{\circ} \mathrm{C}\right)$ storage at initial, 1-month, and 2-month storage time points. No trends were identified for changes in $\mathrm{pH}$ upon storage at either ambient or accelerated conditions. Some inherent variability of cream $\mathrm{pH}$ 


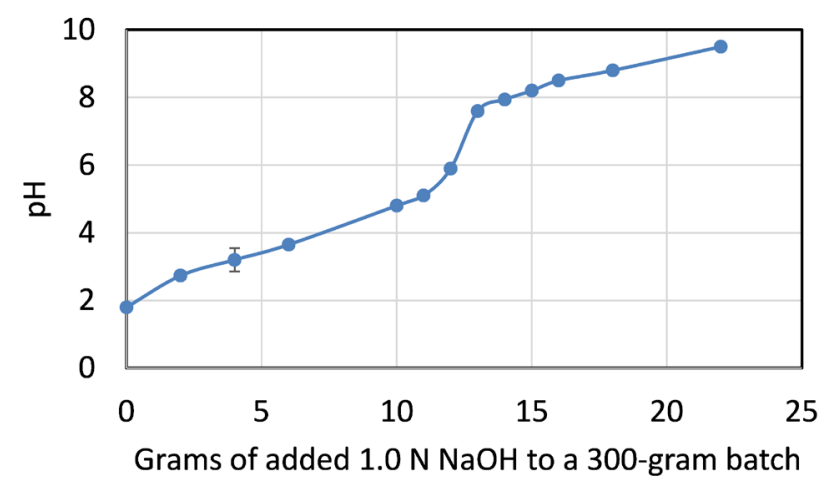

Figure 1. $\mathrm{pH}$ of the separated water phase of the cream as a function of added $1-\mathrm{N} \mathrm{NaOH}$. All measurements were made within 72 hours of cream manufacture. Representative error bars denote \pm 1 standard deviation.

Table 2. $\mathrm{pH}$ of the cream under ambient and accelerated $\left(40^{\circ} \mathrm{C}\right)$ storage conditions.

\begin{tabular}{cccccc}
\hline $\begin{array}{c}1.0-\mathrm{N} \mathrm{NaOH} \\
\text { Added }(\mathrm{g})\end{array}$ & $\begin{array}{c}\text { Initial } \\
\mathrm{pH}\end{array}$ & $\begin{array}{c}\mathrm{pH} \text { 1 month } \\
\text { Ambient }\end{array}$ & $\begin{array}{c}\mathrm{pH} 1 \text { month } \\
40^{\circ} \mathrm{C}\end{array}$ & $\begin{array}{c}\mathrm{pH} 2 \text { month } \\
\text { Ambient }\end{array}$ & $\begin{array}{c}\mathrm{pH} 2 \text { month } \\
40^{\circ} \mathrm{C}\end{array}$ \\
\hline 4.072 & 3.01 & 3.50 & 3.46 & 3.43 & 3.53 \\
6.002 & 3.58 & 3.84 & 3.86 & 3.76 & 3.87 \\
10.115 & 4.80 & 4.40 & 4.92 & 4.66 & 4.73 \\
11.056 & 5.13 & 5.05 & 5.23 & 5.10 & 5.02 \\
12.075 & 5.89 & 5.62 & 5.77 & 5.80 & 5.62 \\
13.045 & 7.58 & 7.37 & 7.43 & 7.43 & 7.23 \\
14.036 & 7.85 & 7.93 & 7.97 & 7.99 & 7.82 \\
15.019 & 8.19 & 8.26 & 8.30 & 8.27 & 8.10 \\
16.027 & 8.46 & 8.50 & 8.57 & 8.58 & 8.38 \\
17.060 & 8.68 & 8.71 & 8.76 & 8.60 & 8.60 \\
18.029 & 8.84 & 9.35 & 8.86 & 8.79 & 8.80 \\
22.028 & 9.45 & 10.10 & 9.45 & 9.40 & 9.43 \\
\hline
\end{tabular}

can be seen in the data, but in general the $\mathrm{pH}$ values before and after storage agreed to within a range of $+/-0.2$, showing the formulation to have a stable shelf life over a reasonable temperature range.

Figure 2 provides as a function of added $\mathrm{NaOH}$ the mass of aqueous phase obtained after centrifugation of $30 \mathrm{~g}$ of cream at 47,500 $\mathrm{g}$ for 2 hours. Results are shown for creams freshly prepared (within $72 \mathrm{hr}$ of production) compared to those stored for 60 days under normal and accelerated conditions. The centrifugation conditions were selected because even the most stable cream centrifuged at $47,500 \mathrm{~g}$ for 2 hours produced a sufficient amount of separated aqueous phase to determine $\mathrm{pH}$. At all $\mathrm{pH}$ values except 8.7, slight differences were observed between the aged and freshly-prepared creams, with a tendency for the creams to become more stable (harder to separate) upon storage. At the $\mathrm{pH}$ value of 8.7, a notable reduction in separated aqueous phase after centrifugation was observed 


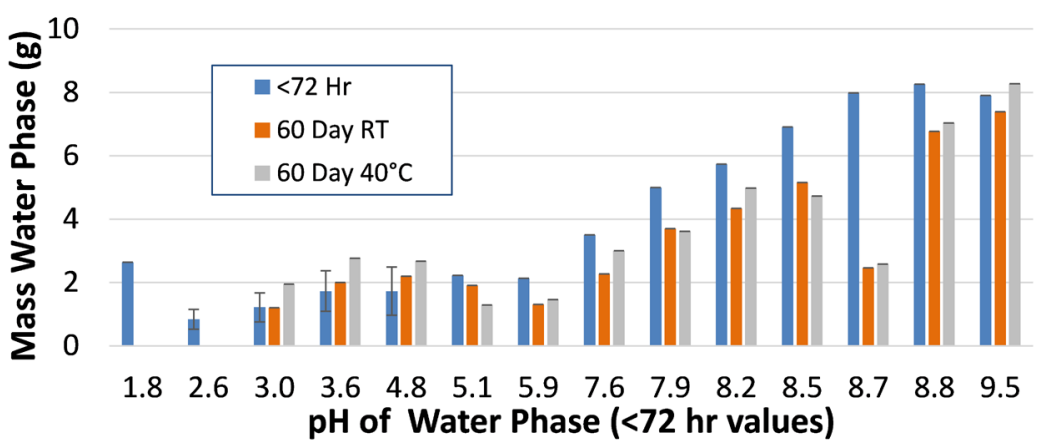

Figure 2. Mass of aqueous phase obtained from fresh and stored samples after centrifugation of 30 grams of cream at 47,500 g for 2 hours. Representative error bars denote \pm 1 standard deviation.

after two months of storage.

Globule size determination for six-week-old creams having $\mathrm{pH}$ values of 4.4, 5.4, 8.4 and 9.2 are shown in Figure 3 with $D_{v} 10, D_{v} 50$, and $D_{v} 90$ values provided in Table 3. As seen, globule size volume distribution is small and uniform with the $\mathrm{CE}$ diameter decreasing with increasing $\mathrm{pH}$. Distribution width is greatest for the cream having $\mathrm{pH}$ value 4.4 compared with the $\mathrm{pH}=9.2$ cream having the narrowest globule size distribution. The two creams having $\mathrm{pH}$ values on either side of the $\mathrm{pH}$ inflection point ( $\mathrm{pH} 5.4$ and 8.4) were very similar in terms of mean globule size and volume distribution.

PLM microphotographs are shown in Figure 4 for a range of $\mathrm{pH}$ values, 4.36 to 9.15. The birefringence patterns show that Crodafos CES emulsions are anisotropic creams at ambient conditions over the entire $\mathrm{pH}$ range. Images of creams having $\mathrm{pH}$ values below 8 exhibit very similar features. When sufficient $\mathrm{NaOH}$ solution has been added to the cream to bring the $\mathrm{pH}$ above the inflection point of the $\mathrm{pH}$ curve seen in Figure 1 (i.e., above $\mathrm{pH}-8.4$ ), the appearance of the PLM images become more inhomogeneous, as shown in the figure for $\mathrm{pH} 9.15$.

\section{Discussion}

The mass of aqueous phase obtained after centrifugation of 30 grams of cream at $47,500 \mathrm{~g}$ for two hours (Figure 2) suggests the presence of a $\mathrm{pH}$ dependence in physical stability of the cream. For $\mathrm{pH}$ values below the inflection point (Figure $1, \mathrm{pH}<6.5$ ), our emulsion is more difficult to break by using centrifugation, as indicated by the lower mass of aqueous phase obtained. Likewise, the decrease in aqueous phase mass after 2 months storage of creams exhibiting $\mathrm{pH}>5.5$ is consistent with an increase in emulsion structure upon storage, at both room temperature and $40^{\circ} \mathrm{C}[10]$. In as much as storage at $40^{\circ} \mathrm{C}$ is an accelerated temperature condition to help predict chemical stability of a product, centrifugation is an accelerated gravity condition to help predict physical stability. The utility of using centrifugation as an accelerated physical stability condition for creams has been described and debated for well over 50 years [11], and in this respect sophisticated ultracentrifugation techniques have shown that in some cases 


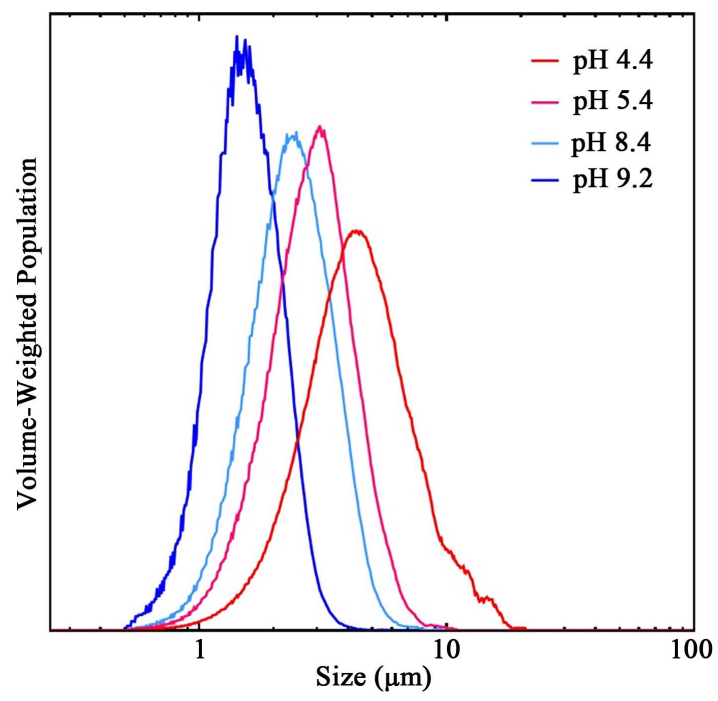

Figure 3. Volume-weighted particle size distributions of Crodafos cream samples (average of three experiments).
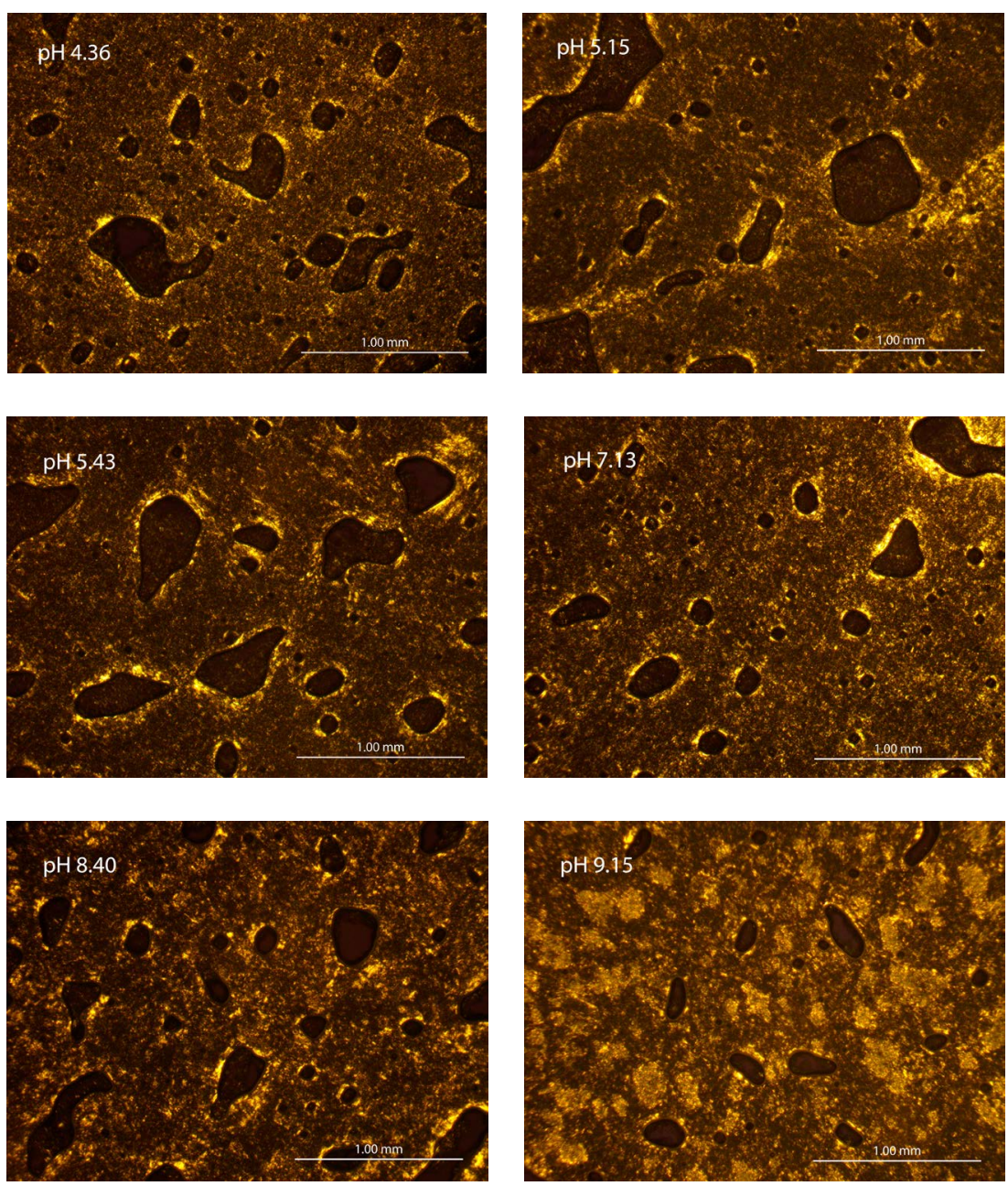

Figure 4. Polarized Microscopic View images of creams at various $\mathrm{pH}$ values between 4.36 and 9.15 . The images were recorded using a $4 \times$ objective. The scale bar in each image corresponds to $1.00 \mathrm{~mm}$. 
Table 3. Globule volume distribution statistics for four measurements of Crodafos CES liquid crystal stabilized creams over a full range of $\mathrm{pH}$ values.

\begin{tabular}{cccc}
\hline pH of Cream & $\mathrm{D}_{\mathrm{V}} 10(\mu \mathrm{m})$ & $\mathrm{D}_{\mathrm{v}} 50(\mu \mathrm{m})$ & $\mathrm{D}_{\mathrm{V}} 90(\mu \mathrm{m})$ \\
\hline 4.4 & 2.3 & 4.3 & 8.2 \\
5.4 & 1.6 & 2.8 & 4.5 \\
8.4 & 1.4 & 2.3 & 3.7 \\
9.2 & 1.0 & 1.5 & 2.3 \\
\hline
\end{tabular}

3-year stability can be accurately predicted after only days of accelerated testing [12]. The data captured in Figure 2 are not offered in an attempt to develop an accelerated testing method but rather as an additional observation related to the formation of stabilizing liquid crystal layers at the oil droplet/water interface.

In a very early systematic review of topical semisolid product microstructure and metamorphosis, Friberg [13] noted that the liquid crystal that stabilizes an emulsion can form spontaneously when its components are simply added to the solvent mixture without the need for energetic mixing. As the liquid crystal structure forms, the oil is spontaneously broken into small droplets to produce a stable colloidal dispersion. This suggests that globule size distributions in this cream may depend significantly on the nature of the emulsifiers (i.e., Crodafos CES neutralization) and not just on the mechanical energy put into the emulsion during processing. Indeed, the gradual shifts we observe to smaller globule volumes and narrower volume distributions as $\mathrm{pH}$ is increased is consistent with our cream exhibiting globule size dependence on composition. However, since mixing time and mixing speed were kept constant in this study, the impact of processing on globule size has yet to be determined.

Blends of the water and oil phases along with the specified amphiphilic compounds in this cream are unique because molecules of the DEGEE solvent are likely to be distributed appreciably throughout the different phases of the cream. Water as the continuous phase is concentrated in those parts of the cream containing the polar groups of the amphiphiles, and the moisturizers (isopropyl palmitate and petrolatum) are concentrated in the hydrophobic internal oil phase. DEGEE, being both miscible in water and a good solvent for the isopropyl palmitate, will be distributed appreciably but non-uniformly throughout the various phases of the cream-the internal oil phase, the liquid crystalline stabilizing layers at the w/o interfaces, and the continuous water phase. This cream formulation will potentially dissolve actives not only in the internal and continuous phases of the emulsion, but also at the liquid crystal stabilized interfaces.

When a liquid crystal stabilized emulsion is placed between the microscope slide and coverslip and viewed using PLM, the image will contain a pattern of birefringence on a dark background. The birefringence results from the threedimensional structured liquid crystals in the cream sample having a diversity of orientations. The plane polarized light entering this anisotropic cream sample is 
rotated to produce a birefringence pattern that is characteristic of the microstructure of the cream. It should be noted that pharmaceutical creams are often isotropic semisolids, thus any cream having a birefringence pattern when viewed using PLM is most likely a liquid crystal stabilized emulsion [14]. The Microscopic View images in Figure 4 are in good agreement with typical PLM appearances of lamellar lyotropic liquid crystalline phases published in the literature [14], which confirms that this cream formulation is a liquid crystal stabilized emulsion over the full topical $\mathrm{pH}$ range of 3.5 to 9 . The mosaic pattern observed in the high $\mathrm{pH}$ creams (Figure 4, $\mathrm{pH}$ 9.15) indicates a shift in microstructure at some point above the $\mathrm{pH}$ value of 8.4 for neutralized Crodafos CES creams. Microscopic View testing of a liquid crystal stabilized emulsion should begin by noting the birefringence pattern of an anisotropic cream and then validating that sample preparation techniques do not significantly alter the image. Changes in the birefringence pattern should then further be monitored over the shelf-life of the product.

For pharmaceutical applications, formulating with an emulsifier that forms stable creams over the $\mathrm{pH}$ range of 2.0 to 10 potentially provides significant practical advantages. As seen in Figure 1, increasing amounts of added $\mathrm{NaOH}$ gradually raises cream $\mathrm{pH}$ up to a value of about 6.0. An inflection point occurs between $\mathrm{pH}$ values of 6 and 8 , before returning to gradually increasing $\mathrm{pH}$ values up to $\mathrm{pH}=10$ with additional neutralization. The $\mathrm{pH}$ range that can be targeted with our Crodafos CES emulsion is thus broader than the typical dermatological product $\mathrm{pH}$ range of 3.5 to 9 . As seen in Table 2, the $\mathrm{pH}$ values of the neutralized Crodafos CES creams did not shift after two months of storage under either ambient or accelerated $\left(40^{\circ} \mathrm{C}\right)$ conditions, implying a lasting shelf life. The broad range of $\mathrm{pH}$ tunability also provides advantages in formulating creams containing ionizable actives. As noted in the Introduction, the degree of ionization for a topically applied active can dramatically affect its skin flux. Thus, our Crodafos CES cream formulation has a broader potential for controlling the ionization of dissolved actives and optimizing skin flux.

\section{Conclusion}

It has been shown that the emulsifying wax blend of cetearyl alcohol, dicetyl phosphate, and ceteth-10 phosphate (tradename Crodafos CES) forms a liquid crystal stabilized oil-in-water emulsion over the $\mathrm{pH}$ range of 3.5 to 9 . This moisturizing cream has white petrolatum and isopropyl palmitate as the oil phase and remains physically stable upon storage at $40^{\circ} \mathrm{C}$ after incorporation of 25 wt\% of DEGEE. Emulsion globule size was dependent on the degree of emulsifying wax neutralization, with the globule size decreasing with increasing cream $\mathrm{pH}$. Since this formulation contains $25 \mathrm{wt} \%$ DEGEE and can be adjusted to any $\mathrm{pH}$ value between 3.5 and 9 , this moisturizing cream vehicle could be modified for optimal skin delivery of a topically applied active pharmaceutical ingredient. 


\section{Conflicts of Interest}

The authors declare no conflicts of interest regarding the publication of this paper.

\section{References}

[1] Osborne, D.W. (2011) Diethylene Glycol Monoethyl Ether: An Emerging Solvent in Topical Dermatology Products. Journal of Cosmetic Dermatology, 10, 324-329. https://doi.org/10.1111/j.1473-2165.2011.00590.x

[2] Loden, M. and Maibach, H.I., Eds. (2000) Dry Skin and Moisturizers: Chemistry and Function. CRC Press, Boca Raton.

[3] Javadzadeh, Y., Adibkia, K. and Hamishekar, H. (2015) Transcutol ${ }^{\circledast}$ (Diethylene Glycol Monoethyl Ether): A Potential Penetration Enhancer. In: Dragicevic, N. and Maibach, H.I., Eds., Percutaneous Penetration Enhancers Chemical Methods in Penetration Enhancement. Modifications of the Stratum Corneum, Springer-Verlag, Berlin, 195-206. https://doi.org/10.1007/978-3-662-47039-8_12

[4] Friberg, S.E., Mandell, L. and Larrson, M. (1969) Mesomorphous Phases, a Factor of Importance for the Properties of Emulsions. Journal of Colloid and Interface Science, 29, 155-156. https://doi.org/10.1016/0021-9797(69)90357-9

[5] Zhang, W. and Liu, L. (2013) Study on the Formation and Properties of Liquid Crystal Emulsion in Cosmetic. Journal of Cosmetics, Dermatological Sciences and Applications, 3, 139-144. https://doi.org/10.4236/jcdsa.2013.32022

[6] US Patent Application 20060204526 "Emulsive Composition Containing Dapsone.” Inventors: Robert W. Lathrop, David W. Osborne.

[7] Prospector Website and Links Therein. https://www.ulprospector.com/en/na/PersonalCare/Detail/134/130780/Crodafos-C $\underline{\mathrm{ES}}$

[8] Li, N., Wu, X., Jia, W., Zhang, M.C., Tan, F. and Zhang, J. (2012) Effect of Ionization and Vehicle on Skin Absorption and Penetration of Azelaic Acid. Drug Delivery and Industrial Pharmacy, 38, 985-994.

https://doi.org/10.3109/03639045.2011.635376

[9] Microscopic View Is a Term Acceptable to the Food and Drug Administration to Describe Using Bright-Field Optical Microscopy to Determine Globule Size or Polarized Light Microscopy for Determining Drug Particle Size for Multi-Phasic Semisolid Products. Microscopic View Testing Is Recommended When Globule Size or Drug Particle Size Is a Q3 Critical Quality Attribute of the Drug Product. https://www.fda.gov/downloads/drugs/newsevents/ucm591915.pdf

[10] The Notably Lower Mass in Figure 2 of the Separated Aqueous Phase from the $\mathrm{pH}$ 8.7 Cream May Be an Artifact and Is Being Further Investigated to Determine Significance.

[11] Garrett, E.D. (1962) Prediction of Stability of Pharmaceutical Preparation VIII Oil-in-Water Emulsion Stability and the Analytical Ultracentrifuge. Journal of Pharmaceutical Sciences, 51, 35-42. https://doi.org/10.1002/jps.2600510105

[12] Andre, V., Willenbacher, N., Debus, H., Borger, L., Fernandez, P., Frechen, T. and Rieger, J. (2003) Prediction of Emulsion Stability: Facts and Myth. Cosmetics and Toiletries Manufacture Worldwide, 102-109.

[13] Friberg, S.E. (1990) Micelles, Microemulsions, Liquid Crystals and the Structure of the Stratum Corneum Lipids. Journal of the Society of Cosmetic Chemists, 41, $155-171$ 
[14] Ekwall, P. (1975) Composition, Properties and Structures of Liquid Crystalline Phases in Systems of Amphiphilic Compounds. In: Brown, G.H., Ed., Advances in Liquid Crystals, Vol. 1, Academic Press, New York, 1-142.

https://doi.org/10.1016/B978-0-12-025001-1.50007-X 